Physical Activity and Nutrition I 2021;25(3):008-015

Received: 2021/08/16, Revised: 2021/09/05, Accepted: 2021/09/06, Published: 2021/09/30 @2021 Aki Kawamura et al.; Licence Physical Activity and Nutrition. This is an open access article distributed under the terms of the creative commons attribution license (https:/l creativecommons.org/licenses/by-nc/2.0/), which permits unrestricted use, distribution, and reproduction in any medium, provided the orginal work is properly cited.

${ }^{*}$ Corresponding author: Masaaki Sugita, Ph.D.

Faculty of Sport Science, Nippon Sport Science University, 7-1-1, Fukasawa, Setagaya-ku, Tokyo, 158-8508, Japan

Tel: +81-03-5706-1148 / Fax: +81-03-5706-1148

E-mail: m-sugita@nittai.ac.jp

@2021 The Korean Society for Exercise Nutrition

\section{Oligomerized polyphenols in lychee fruit extract supplements may improve high-intensity exercise performance in male athletes: a pilot study}

\author{
Aki Kawamura ${ }^{1}$ / Shun Hashimoto ${ }^{1}$ / Miho Suzuki ${ }^{2}$ \\ Hiromasa Ueno $^{3,4}$ / Masaaki Sugita ${ }^{2 *}$ \\ 1. Sports Science Research Promotion Centre, Nippon Sport Science University, Tokyo, Japan \\ 2. Faculty of Sport Science, Nippon Sport Science University, Tokyo, Japan \\ 3. Graduate School of Health and Sport Science, Nippon Sport Science University, Tokyo, Japan \\ 4. Research Fellow of Japan Society for the Promotion of Science, Tokyo, Japan
}

\section{INTRODUCTION}

induced by prolonged high-intensity exercise can cause structural and functional damage. Antioxidant polyphenol supplementation, which reduces ROS levels, may improve high-intensity exercise performance. We evaluated the effect of lychee fruit extract, which contains high levels of low-molecular-weight oligomerized polyphenols, on high-intensity exercise performance.

[Methods] Ten male athletes were included in an open-label trial that consisted of control and intervention phases, with a 7-day washout period between phases. The participants were administered oligomerized lychee fruit extract for seven days, whereas no intervention was given in the control phase. High-intensity intermittent exercise and the Wingate test were performed. The power output, blood lactate levels, reactive oxygen metabolite levels, biological antioxidant potential, heart rate, and rate of perceived exertion were measured.

[Results] The average power output was significantly higher in the intervention phase than in the control phase $(P<0.01)$, while the change in blood lactate levels was significantly lower in the intervention phase than in the control phase $(P<0.05)$. The average heart rate was significantly higher in the intervention phase than in the control phase $(P<0.05)$, without changing the rate of perceived exertion. Although there was no difference in reactive oxygen metabolite levels between the phase, the change in biological antioxidant potential was larger in the intervention phase than in the control phase $(P=0.06)$. The Wingate test showed no significant differences between the phase.

[Conclusion] Short-term loading with oligomerized lychee fruit extract may increase performance during high-intensity intermittent exercise by improving metabolism.

[Key words] antioxidant capacity, fruit polyphenols, oligomerized lychee fruit extract, high-intensity intermittent exercise, power output, reactive oxygen metabolites
Exercise-induced reactive oxygen species (ROS) act as signaling factors for physiological adaptations and pro-inflammatory responses. Exercise patterns, such as intensity and duration, affect ROS generation ${ }^{1}$. Short-duration exercise intermittently produces low to moderate levels of ROS, activates signaling pathways, and promotes cellular adaptations, all of which exert a protective effect against subsequent stress ${ }^{2}$. Conversely, high levels of ROS induced by prolonged or high-intensity exercise may cause structural and functional damage ${ }^{3}$. In particular, excessive ROS production during strenuous exercise impairs physiological functions, such as blood flow-mediated vasodilation and muscle contraction ${ }^{4-6}$. $\mathrm{Reid}^{4}$ showed that redox interventions are important factors in exercise performance. ROS are generated during high-intensity exercise as a consequence of increased aerobic metabolism ${ }^{7-9}$. Excessive ROS may cause oxidative damage to tissues and cells ${ }^{10}$ and decrease muscle function ${ }^{5,6}$. Highly trained athletes perform high-intensity exercises and are exposed to high levels of ROS, which may lead to physical dysfunction and a decline in performance ${ }^{4,11}$. In addition to the high levels of ROS, antioxidant defense systems, such as enzymes (superoxide dismutase, catalase, glutathione peroxidase, etc.) and exogenous antioxidants (vitamins, polyphenols, etc.) are activated. Therefore, it would be beneficial for athletes to take adequate amounts of antioxidants during high-intensity exercise to enhance the capacity of exogenous antioxidants.

However, there is inconsistent evidence on whether antioxidant supplementation improves athletic performance ${ }^{12}$. Previous studies have shown that long-term (10 to 11 weeks) high-dose vitamin $\mathrm{C}$ and $\mathrm{E}$ intervention negatively ${ }^{13}$ or ineffectively ${ }^{14}$ affects muscle performance and may induce maximal voluntary contraction. Although vitamin $\mathrm{C}$ effectively suppressed inflammation and muscle damage via short-term intervention ${ }^{15}$, a recent systematic review confirmed that vitamins $\mathrm{C}$ and E did not have a performance-modulating effect ${ }^{12,16}$.

In contrast, low to moderate doses of food-derived polyphenols improve muscular function in long-term $(10 \text { weeks })^{17}$ and short-term (7 to 
8 days) interventions through their antioxidant capacity ${ }^{18-22}$. These results suggest that the effect of antioxidants on exercise adaptation depends on the type and dose of antioxidants administered. In particular, an appropriate amount of food-derived polyphenol supplementation may benefit muscle performance, even with short-term loading ${ }^{23}$. However, negative or ineffective responses that interfere with exercise adaptation may occur due to the administration of wrong dosages or durations and low bioavailability of antioxidants $^{24,25}$.

Generally, polyphenols have low in vivo bioavailability due to several factors, such as their interaction with the food matrix during digestion and metabolic processes mediated by the liver, intestine, and microbiota ${ }^{26}$. However, oligomerized lychee fruit extract (OLFE) is manufactured as a low-molecular-weight polyphenol and has higher bioavailability and stronger antioxidant capacity because it is hydrolyzed and stabilized at a smaller molecular size through manufacturing. Indeed, serum polyphenol levels increase upon in vivo intervention with OLFE supplementation compared to lychee fruit intake ${ }^{27}$. In addition, OLFE suppresses ROS production and inflammation in humans and animals ${ }^{28-30}$. These properties enable safe and effective low-dose antioxidant supplementation, which may contribute to performance improvement in various exercise-related scenarios.

In the athletic field, high-intensity exercise-induced inflammation is significantly reduced after a $100 \mathrm{mg} /$ day OLFE intervention for two months in male endurance athletes $^{31}$ and four weeks in healthy young men ${ }^{28}$. Tsukamoto et al. showed that supplementation with OLFE at a $200 \mathrm{mg} /$ day dose for two weeks elevates the maximum oxygen uptake in 20 sets of high-intensity intermittent exercise (HIIE) in male athletes ${ }^{32}$. Another study showed that supplementation with the same dose for 26 days decreased subjective fatigue in athletes after training ${ }^{33}$. These results suggest that OLFE supplementation from 100 to $200 \mathrm{mg}$ /day enhances aerobic capacity and reduces exercise-induced inflammation without harmful effects.

HIIE requires both aerobic and anaerobic capacities. It is applied in various sports, repeating high-intensity exercise intermittently with short-term recovery or low-intensity exercise in between ${ }^{34}$. At the beginning of HIIE, anaerobic energy metabolism, such as ATP-PCr and the glycolysis cycle, dominates aerobic energy metabolism. In contrast, aerobic energy metabolism dominates as exercise duration increas$\mathrm{es}^{35,36}$.

In this study, we hypothesized that supplementation with OLFE may improve HIIE performance after seven days. Therefore, we conducted a pilot study to evaluate the safety and efficacy of OLFE intake on exercise performance in athletes.

\section{METHODS}

\section{Participants}

Ten male athletes from a university sports club specializing in intermittent exercise (age: $21.5 \pm 0.8 \mathrm{y}$, height: $171.7 \pm 4.1 \mathrm{~cm}$, weight: $69.2 \pm 6.4 \mathrm{~kg}$ ) were recruited. The ethics committee of Nippon Sport Science University (No. 019-H045) and the University Hospital Medical Information Network Clinical Trials Registry in Japan (No. UMIN000041049) approved this study. After being informed of the purpose of the study, methods, possible health hazards, risks, privacy protection, data management, and publication, all participants signed a consent form. None of the participants had an ongoing or prior chronic disease or a history of smoking before enrolment in this study.

All participants were instructed to avoid using supplements and medicines that could affect OLFE supplementation during the intervention period. The body composition of the participants was measured using bioelectrical impedance analysis (InBody 730, InBody Co., Ltd., Seoul, Korea), and their physical characteristics are shown in Table 1. Participants trained five to six times a week, and their average maximum power output for the Wingate test at the baseline was $10.9 \pm 0.9 \mathrm{~W} / \mathrm{kg}$. As 9 out of 10 participants competed in the same sports club and had similar training regimes, there was no significant difference in the training

Table 1. Physical characteristics of participants in CON and OLI trial.

\begin{tabular}{|c|c|c|c|c|c|c|c|c|}
\hline \multirow[t]{2}{*}{ Participants } & \multirow{2}{*}{$\begin{array}{c}\text { Age } \\
\text { (years) }\end{array}$} & \multirow{2}{*}{$\begin{array}{l}\text { Height } \\
(\mathrm{cm})\end{array}$} & \multicolumn{2}{|c|}{$\begin{array}{l}\text { Body weight } \\
\text { (kg) }\end{array}$} & \multicolumn{2}{|c|}{$\begin{array}{c}\text { BMI } \\
\left(\mathrm{kg} / \mathrm{m}^{2}\right)\end{array}$} & \multicolumn{2}{|c|}{$\begin{array}{c}\text { Body fat } \\
(\%)\end{array}$} \\
\hline & & & CON & OLI & CON & OLI & CON & OLI \\
\hline a & 22 & 178.5 & 70.9 & 70.2 & 22.3 & 22.0 & 13.6 & 14.3 \\
\hline$b$ & 22 & 168.0 & 57.8 & 58.3 & 20.5 & 20.7 & 8.1 & 8.4 \\
\hline c & 21 & 168.0 & 60.8 & 60.3 & 21.5 & 21.4 & 14.5 & 13.4 \\
\hline d & 21 & 168.0 & 70.8 & 69.6 & 25.1 & 24.7 & 11.1 & 10.0 \\
\hline e & 23 & 176.0 & 78.8 & 77.0 & 25.4 & 24.9 & 17.0 & 15.8 \\
\hline$f$ & 20 & 177.7 & 74.6 & 74.5 & 23.6 & 23.6 & 12.1 & 11.5 \\
\hline g & 22 & 171.0 & 71.6 & 70.1 & 24.5 & 24.0 & 15.4 & 15.2 \\
\hline $\mathrm{h}$ & 22 & 173.0 & 63.7 & 65.7 & 21.3 & 22.0 & 11.0 & 10.5 \\
\hline i & 21 & 170.2 & 67.2 & 66.7 & 23.2 & 23.0 & 15.4 & 15.4 \\
\hline j & 21 & 167.0 & 76.0 & 76.0 & 27.3 & 27.3 & 19.4 & 19.6 \\
\hline Mean & 21.5 & 171.7 & 69.2 & 68.8 & 23.5 & 23.3 & 13.8 & 13.4 \\
\hline SD & 0.8 & 4.1 & 6.4 & 5.9 & 2.0 & 1.9 & 3.1 & 3.2 \\
\hline
\end{tabular}

$n=10$, SD; standard deviation, BMI; body mass index, CON; control-intervention, OLI; oligomerised lychee fruit extract-intervention, age and height were measured at baseline. Body weight, BMI, and body fat were measured before each trial. 
load between participants and phases during the intervention period. In addition, there were no official matches or other athletic events that took place during the intervention period.

\section{Experimental design}

This study was designed as an open-label trial consisting of control intervention (CON) and OLFE intervention (OLI) phases. All participants practiced before the trial to confirm the experimental protocol. A washout period of 7 days was set between the CON and OLI phases. All participants refrained from high-intensity exercise and drinking alcohol one day before the trial. They had the same meals at the same time and avoided caffeine and alcohol on the trial day. Body composition and subjective fatigue were assessed pre-trial for the CON and OLI phases. The degree of subjective fatigue was measured before exercise (at rest) using a visual analog scale method, and the participants were asked to indicate the degree on a $100 \mathrm{~mm}$ horizontal line.

The participants performed a warm-up exercise for 15 min with a load of $1.0 \mathrm{kpc}$ at $60 \mathrm{rpm}$ using a bicycle ergometer (Fujin Raijin, Ohashi Chiso Laboratory Co., Ltd., Japan). After sitting for $20 \mathrm{~min}$, HIIE was performed at a load of $7.5 \%$ of the bodyweight for 10 sets. The subjects rested for $30 \mathrm{~min}$, and then a Wingate test was performed for $30 \mathrm{~s}$ under the same load. Power output, blood lactate levels, levels of reactive oxygen metabolites (diacron-reactive oxygen metabolites [d-ROMs]), biological antioxidant potential (BAP), heart rate, and rate of perceived exertion (RPE) were measured before, during, and after exercise (Fig. 1). The participants were allowed to drink water during the trial freely.

\section{Supplementation}

Oligonol (Amino Up Chemical Co., Ltd., Sapporo, Japan) supplement extracted from lychee fruit pericarp, which was mechanically oligomerized to polyphenol polymers, was used. It contains low-molecular-weight oligomerized polyphenols (100 mg per capsule). In addition, $15.7 \mathrm{w} / \mathrm{w} \%$ polyphenol monomers $((+)$-catechin and (-)-epicatechin) and $13.3 \mathrm{w} / \mathrm{w} \%$ polyphenol dimers (procyanidin B2) were also

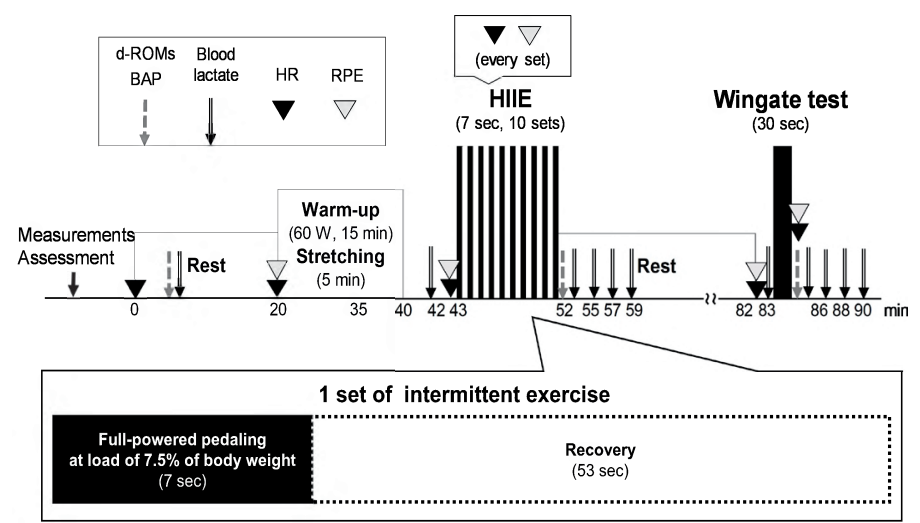

Figure 1. Experimental protocol. HIIE, high-intensity intermittent exercise; d-ROMs, reactive oxygen metabolites; BAP, biological antioxidant potential; $\mathrm{HR}$, heart rate; RPE, rate of perceived exertion. mixed to stabilize the capsule. All participants were administered with two capsules of OLFE per day, after breakfast and dinner, for seven days in the OLI phase. The total daily intake of polyphenols was $200 \mathrm{mg}$, as reported previously ${ }^{33}$.

\section{Power output measurement}

During the HIIE and the 30-s Wingate test, power output was calculated every $0.1 \mathrm{~s}$ using a bicycle ergometer. One HIIE set consisted of full-powered pedaling for $7 \mathrm{~s}$ at a load of $7.5 \%$ body weight, followed by 53 s of recovery. The exercise was repeated in ten sets. The average power output was calculated for each set and corrected according to body weight. The Wingate test was performed for $30 \mathrm{~s}$ with a load of $7.5 \%$ of the body weight. The average power output, maximum power output, and rate of power decline over a 30 -s period were calculated and corrected according to body weight.

\section{Blood biomarker measurements}

Briefly, $100 \mu \mathrm{L}$ of blood was collected from the fingertip to measure blood lactate, d-ROMs, and BAP. Blood lactate levels were measured using a lactate analyzer (Lactate Pro2, ARKRAY, Inc., Kyoto, Japan) at rest, before exercise, immediately after exercise, and at 3, 5, and 7 min after exercise. The highest value of blood lactate immediately and at 3 , 5 , and $7 \mathrm{~min}$ after exercise was selected as the blood lactate level (mmol/L) after exercise. Blood samples were collected in heparinized vacuum tubes (Microvette, SARSTEDT AG \& Co. KG, Nümbrecht, Germany) and centrifuged at 2,000 $\times \mathrm{g}$ at room temperature for $2 \mathrm{~min}$.

Plasma samples were used to measure d-ROMs and BAP using a free radical analyzer (FREE Carrio Duo, Diacron International s.r.1., Grosseto, Italy) $)^{37,38}$ at rest and immediately after each exercise.

For d-ROM (U.CARR) measurements, $20 \mu \mathrm{L}$ of plasma was added to a mixture containing an acidic buffer solution (pH 4.8) and an aqueous aromatic amine solution. A chromogen was added to the mixed sample and transferred to a spectrophotometer. The plasma hydroperoxide concentration was calculated from the percentage absorbance change at $505 \mathrm{~nm}$ during $5 \mathrm{~min}$ of incubation, which estimated the d-ROM (U.CARR) levels

BAP $(\mu \mathrm{mol} / \mathrm{L})$ was measured via the change in the concentration of ferric ions $\left(\mathrm{Fe}^{3+}\right)$ to ferrous ions $\left(\mathrm{Fe}^{2+}\right)$ reduced by the endogenous antioxidants and the exogenous antioxidants in the blood ${ }^{39}$. Briefly, $10 \mu \mathrm{L}$ of plasma was added to the reaction mixture, and the absorbance was measured at $505 \mathrm{~nm}$. The sample was incubated at $37{ }^{\circ} \mathrm{C}$ for $5 \mathrm{~min}$, and the absorbance at $505 \mathrm{~nm}$ was measured again. The percentage change in absorbance was calculated from the oxidized iron ion concentration, which provided an estimated BAP.

\section{Heart rate and RPE}

Heart rate (bpm) was measured every second using a heart rate sensor (H10, POLAR M200, POLAR, Polar Electro, Kempele, Finland). Maximum values were set at each minute of HIIE and $30 \mathrm{~s}$ of full-powered exercise. The RPE consisted of 15 levels (6-20) of the Borg scale and was 
measured immediately after warming up, before, during, and after each exercise.

\section{Statistical analysis}

All data are presented as the mean \pm standard deviation (SD). Differences between the CON and OLI phases were compared using the paired $t$-test or two-way repeated-measures analysis of variance (ANOVA). When significant differences were determined using ANOVA, post hoc analyses were conducted using the Bonferroni test. Nonparametric statistical analysis was performed for parameters without normality. Cohen's $d$ was calculated to measure the effect size between the control and intervention groups. Statistical analysis was performed using SPSS ver.25 (IBM Japan Inc., Tokyo, Japan), and a $P$-value $<0.05$ was considered statistically significant.

\section{RESULTS}

\section{Pre-trial findings}

We found no significant differences in body weight $(\mathrm{kg})$, body mass index, body fat (\%), perception of fatigue (mm) (CON: 75.6 \pm 6.9, OLI: $74.0 \pm 12.8$ ), and sleep duration (hr) (CON: $6.9 \pm 1.7$, OLI: $6.7 \pm 1.4$ ) between the CON and OLI phases. The average room temperature $\left({ }^{\circ} \mathrm{C}\right)(\mathrm{CON}: 23.7 \pm$ 1.0, OLI: $23.6 \pm 0.8)$ and humidity (\%) (CON: $36.1 \pm 5.8$, OLI: $36.8 \pm 5.6$ ) were not different.

\section{Power output}

The average power output $(\mathrm{W} / \mathrm{kg})$ of sets $1,2,4,7$, and 8 was significantly higher in the OLI phase than that in the CON phase $(P<0.05)$ (Fig. 2a), and the average power output $(\mathrm{W} / \mathrm{kg})$ of ten sets in the OLI phase $(9.8 \pm 0.6)$ was significantly higher than that in the CON phase $(9.6 \pm 0.6)$ during HIIE $(P<0.01, d=0.40)$ (Fig. 2b).

In addition, the average power output (W/ $/ \mathrm{kg}$ ) of the 30 -s Wingate test did not differ between the $\mathrm{CON}(8.9 \pm 0.8)$ and OLI ( $8.7 \pm 0.7)$ phases (Fig. 3). Furthermore, the maximum power output $(\mathrm{W} / \mathrm{kg})(\mathrm{CON}: 11.2 \pm 1.1, \mathrm{OLI}: 11.3 \pm 0.9)$ a

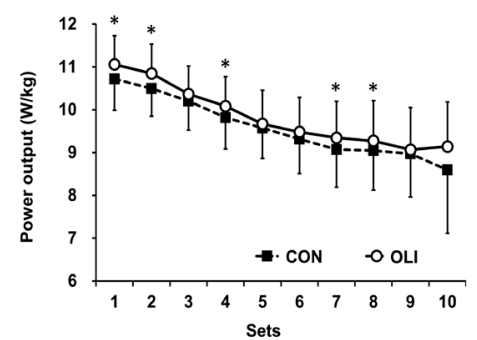

b

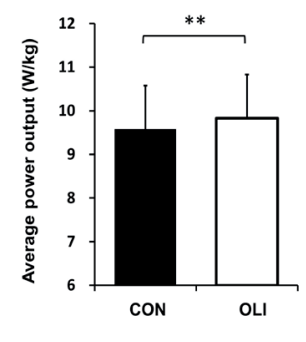

Figure 2. Comparison of the average power output during HIIE. Data are presented as the mean $\pm S D, n=10$. Changes in power output during HIIE for each set (a) and the average of ten sets (b). CON, control intervention; OLI, oligomerized lychee fruit extract-intervention; HIIE, high-intensity intermittent exercise. *Significant difference between CON and OLI at $P<0.05,{ }^{*}$ Significant difference between CON and OLI at $P<0.01$.

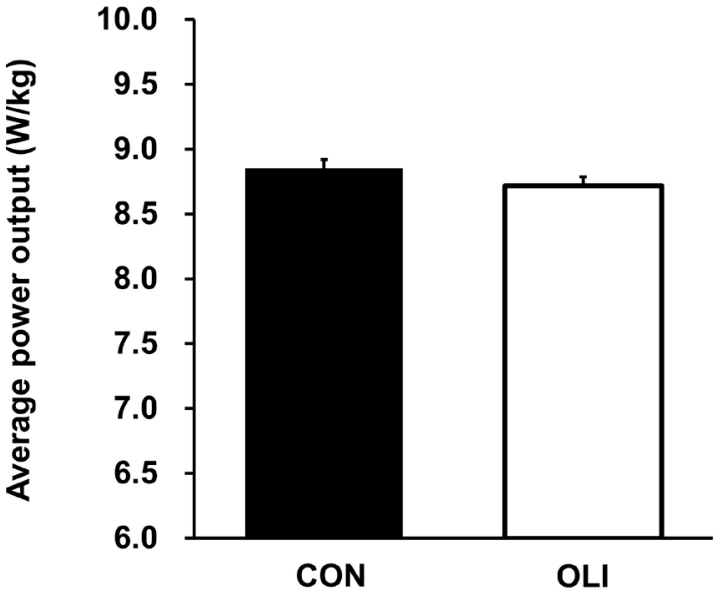

Figure 3. Comparison of the average power output in the 30 -s Wingate test. Data are presented as the mean $\pm S D, n=10$. CON, control intervention; OLI, oligomerized lychee fruit extract intervention.

and the rate of power decline (\%) (CON: $45.9 \pm 13.2$, OLI: $47.1 \pm 12.6$ ) during the 30 -s Wingate test were also not different between the CON and OLI phases.

\section{Blood lactate levels}

The blood lactate level $(\mathrm{mmol} / \mathrm{L})$ was not significantly different between CON and OLI phases before (CON: $1.1 \pm$ 0.1 , OLI: $1.2 \pm 0.4$ ) or after (CON: $13.8 \pm 2.4$, OLI: $12.7 \pm$ 2.5) HIIE. However, the change (\%) was significantly lower in the OLI phase $(1078.9 \pm 258.1)$ than that in the CON phase $(1270.6 \pm 291.0)$ in HIIE $(P<0.05, d=0.70)$ (Fig. 4).

Similarly, the blood lactate level $(\mathrm{mmol} / \mathrm{L})$ was not significantly different between the CON and OLI phases before $(\mathrm{CON}: 6.3 \pm 2.3$, OLI: $6.1 \pm 1.6)$ and after (CON: $14.9 \pm$ 2.4 , OLI: $14.3 \pm 2.0)$ the Wingate test, and the change (\%) was not different between the CON $(266.4 \pm 85.7)$ and OLI $(245.8 \pm 47.2)$ phases.

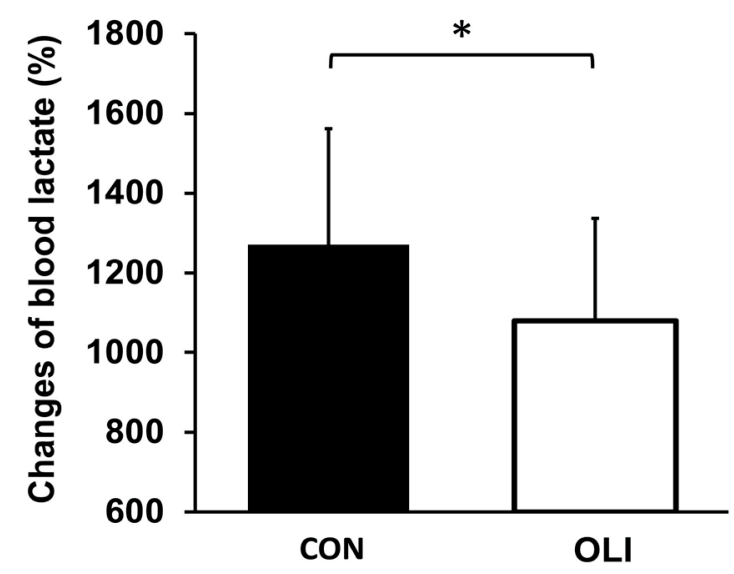

Figure 4. Comparison of the changes in blood lactate levels before and after HIIE. Data are presented as the mean $\pm S D, n=10$. CON, control intervention; OLI, oligomerized lychee fruit extract intervention; HIIE, high-intensity intermittent exercise. *Significant difference between CON and OLI at $P<0.05$. 


\section{ROS production and antioxidant capacity}

The level of d-ROMs (U.CARR) did not differ between the CON and OLI phases before (CON: $286.3 \pm 47.3$, OLI: $283.9 \pm 47.2)$ and immediately after (CON: $313.5 \pm 55.8$, OLI: $310.0 \pm 53.0) \mathrm{HIIE}$, and there was no difference in the change (\%) between the CON $(109.4 \pm 41.8)$ and OLI (109.5 \pm 9.1 ) phases (Fig. 5a). Also, d-ROM (U.CARR) was not significantly different between the CON and OLI phases before (CON: $286.3 \pm 47.3$, OLI: $283.9 \pm 47.2)$ and after (CON: $301.7 \pm 40.8$, OLI: $299.1 \pm 55.2)$ the Wingate test, and the change (\%) was not different between phases (CON, 106.0 \pm 4.7 ; OLI, $105.3 \pm 5.9$ ).

Regarding BAP $(\mu \mathrm{mol} / \mathrm{L})$, there was no significant difference between the CON and OLI phases before (CON: $2085.9 \pm 143.6$, OLI: $2006.2 \pm 205.4)$ and immediately after (CON: $2658.3 \pm 241.9$, OLI: $2698.5 \pm 180.1$ ) HIIE. However, the change in BAP (\%) tended to be higher in the OLI phase $(135.3 \pm 10.0)$ than in the CON phase $(127.4 \pm$ 6.2) during HIIE ( $P=0.06, d=1.22)$ (Fig. 5b). Also, BAP $(\mu \mathrm{mol} / \mathrm{L})$ was not significantly different between phases before (CON: $2085.9 \pm 143.6$, OLI: $2006.2 \pm 205.5)$ and after (CON: $2631.9 \pm 213.9$, OLI: $2626.0 \pm 229.7$ ) the Wingate test and the change (\%) was not different between phases (CON, $126.4 \pm 9.0$; OLI, 131.9 \pm 15.0 ).

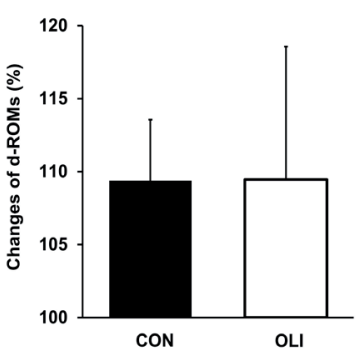

b

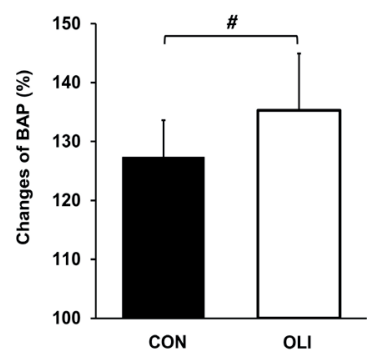

Figure 5. Comparison of the changes in d-ROMs and BAP before and after HIIE. Data are presented as the mean $\pm S D, n=10$. The changes in d-ROMs (a) and BAP (b) before and after HIIE. $\mathrm{d}$-ROMs, reactive oxygen metabolites; BAP, biological antioxidant potential; CON, control intervention; OLI, oligomerized lychee fruit extract intervention; HIIE, high-intensity intermittent exercise. \#Difference at $P=0.06$ compared with $\mathrm{CON}$.

\section{Heart rate and RPE}

The maximum heart rate (bpm) after 2, 3, 4, 7, 8, 9, and 10 sets were significantly higher in the OLI phase than in the CON phase $(P<0.05, P<0.01)$ (Fig. 6). Furthermore, the average heart rate (bpm) after ten sets of HIIE was also significantly higher in the OLI phase $(160.3 \pm 10.9)$ than in the CON phase $(155.9 \pm 10.8)(P<0.05, d=0.40)$. In the Wingate test, the maximum heart rate (bpm) and the average heart rate (bpm) were not significantly different between the CON (168.1 \pm 8.2 and $144.6 \pm 8.5$, respectively) and OLI (167.0 \pm 10.9 and $147.5 \pm 10.1$, respectively) phases.

We observed that the RPE was not different in each HIIE set, and the mean RPE values of the ten sets did not differ between the CON $(16.6 \pm 1.6)$ and OLI (16.9 \pm 1.2$)$ phases. Furthermore, the RPE immediately after the Wingate test

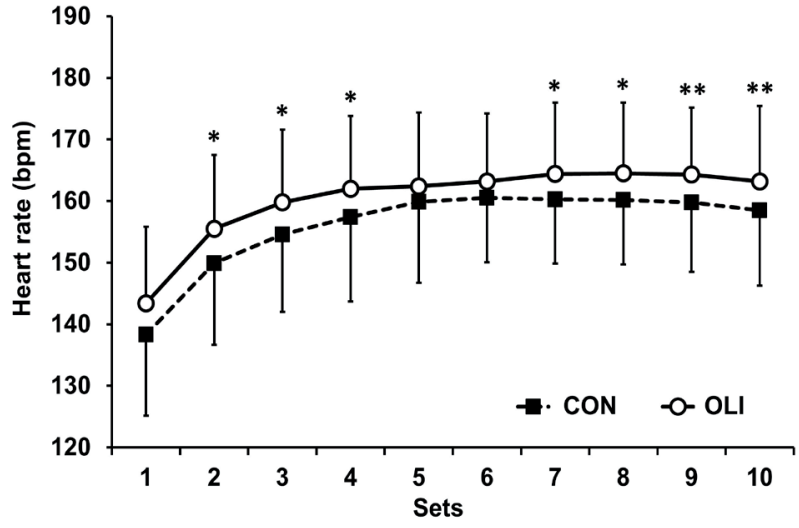

Figure 6. Comparison of the maximum heart rate during HIIE. Data are presented as the mean $\pm S D, n=10$. CON, control intervention; OLI, oligomerized lychee fruit extract intervention; HIIE, high-intensity intermittent exercise. *Significant difference between CON and OLI at $P<0.05,{ }^{*}$ Significant difference between CON and $\mathrm{OLI}$ at $P<0.01$.

was not significantly different between the CON $(18.9 \pm 1.5)$ and OLI $(19.3 \pm 1.2)$ phases.

\section{DISCUSSION}

Our study revealed that seven days of OLFE supplementation positively affected HIIE performance in the trained athletes. The power output was significantly increased in the first, second, and latter sets of HIIE in the OLI phase, which suggested that short-term OLFE loading might improve HIIE performance. Although the sample size was small and it was difficult to obtain a significant difference, short-term OLFE loading resulted in a small but significant change in exercise performance. In addition, the RPE did not change between the CON and OLI phases, which suggested that the participants' subjective exercise intensity did not increase with power output after OLFE supplementation.

With respect to energy metabolism, anaerobic metabolism was dominant in the first and second sets, and the ratio of aerobic metabolism was increased in the subsequent sets $^{36,40}$. Therefore, OLFE loading might contribute to an increase in power output via improved anaerobic and aerobic metabolism. The heart rate was also significantly higher during HIIE in the OLI group, which indicated that the increased power output resulted in a higher heart rate. In parallel, the reduction of blood lactate after exercise in the OLI phase could be interpreted as an improvement in aerobic capacity by OLFE during HIIE.

In the present study, OLFE loading increased oxygen uptake during HIIE, as heart rate is a factor in calculating oxygen uptake according to the Fick equation ${ }^{41}$. Indeed, previous studies have shown that OLFE supplementation increases the maximum oxygen uptake during $\mathrm{HIIE}^{32}$ and extends the time to exhaustion to $80 \%$ with the maximum heart rate ${ }^{42}$. These results suggest that OLFE supplementation can enhance aerobic capacity, and our data indirectly 
support this hypothesis. The mechanism of enhancing aerobic capacity by OLFE is not yet elucidated; however, some food-derived polyphenols have been shown to activate mitochondrial biogenesis and function in skeletal muscles ${ }^{43,44}$. Changes in blood lactate levels were significantly lower in the OLI phase despite the increased power output and heart rate during HIIE. OLFE supplementation has been shown to suppress lactate production through mitochondrial adaptation ${ }^{45,46}$ but further studies are warranted to clarify the effects of OLFE at the molecular level.

Previous studies have shown that ROS production increases with increased exercise intensity ${ }^{7-9}$. Furthermore, the levels of metabolites and d-ROM were significantly elevated after high-intensity exercise compared with those at rest ${ }^{47}$. Here, we expected d-ROM levels to increase since power output increased after OLFE supplementation; however, the changes before and after exercise in the OLI phase were not different from those in the CON phase. These results suggest that OLFE modulates exercise-induced ROS. However, the change in BAP tended to increase after exercise with OLFE supplementation. Although there were no significant changes in BAP between the CON and OLI phases, the trend suggested that blood polyphenol concentrations increased after $7 \mathrm{~d}$ of supplementation with $\mathrm{OLFE}^{27}$, reinforcing its antioxidant potential. In addition, Arent et al. 48 reported that supplementation with a food-derived polyphenol extract during HIIE with an ergometer significantly improved antioxidant properties compared to a placebo 30 and $60 \mathrm{~min}$ after exercise, not immediately after exercise. A significant increase in BAP was not observed because the measurement was performed only immediately after exercise in this study.

Previous studies have shown that the power output during high-intensity exercise increases upon acute ${ }^{49}$ or 7 to 8 -day ${ }^{18,19}$ supplementation of polyphenol-rich fruits in trained males. Studies have also shown that polyphenol-rich fruits attenuate inflammation and muscle soreness during high-intensity exercise. Similarly, OLFE supplementation reduces high-intensity exercise-induced inflammation ${ }^{28,29}$ and muscle damage ${ }^{31}$. Although the mechanism of action of these polyphenols remains unclear, their antioxidant potential might support training-induced inflammation.

The effects of OLFE supplementation on athletic performance have not been previously investigated. In the present study, power output during HIIE increased after seven days of supplementation with $200 \mathrm{mg}$ OLFE, suggesting that short-term OLFE loading improves athletic performance. In particular, the regulation of cytosolic calcium homeostasis is a possible factor for the improvement of power output during exercise sets. Intracellular calcium concentration is regulated by calcium released from the endoplasmic reticulum in muscle cells. When the muscle is stimulated, calcium is released from the sarcoplasmic reticulum into the muscle cytoplasm through a calcium channel, the ryanodine receptor ${ }^{50}$. The muscle then contracts through the activation of actin-myosin interactions, and the ryanodine receptor is post-translationally modified by ROS. Since excessive ROS may prevent calcium release and attenuate muscle contrac- tion $^{6}$, OLFE supplementation may prevent the oxidative modification of the receptor via antioxidant effects ${ }^{30}$. Therefore, the increase in power output in the first and second sets of HIIE in this study might be due to the antioxidant potential of OLFE, which might reduce ROS generation.

Furthermore, OLFE supplementation did not impair post-exercise performance (30-s Wingate test). It was estimated that the physical load increased according to the power output increase during HIIE, which attenuated post-exercise performance. However, this result was not expected. This result suggests that the antioxidant potential of OLFE might suppress post-exercise inflammation and promote recovery ${ }^{28,29,31}$. Therefore, the present study suggests that OLFE supplementation may improve HIIE performance without negative post-exercise outcomes.

This pilot study has both strengths and limitations. Its strengths include excellent adherence of participants to the intervention, with no dropouts. In contrast, there are some limitations, particularly the use of a small group of participants and the lack of a placebo group. Although the results of this open-label study are encouraging, future trials need to be designed, including a placebo group with a crossover design, to validate the effects of OLFE on high-intensity exercise performance. In addition, we were not able to conduct a dietary survey during the intervention phases, although participants were instructed to avoid supplements and antioxidants or changes to their diet.

Another limitation is that we did not measure d-ROMs and BAP during HIIE since we could not measure the changes in d-ROMs and BAP in each set during HIIE. As mentioned above, the physical exertion of the first and second sets of HIIE might be related to the antioxidant potential of OLFE. Further studies are required to measure oxidative stress and the antioxidant potential of OLFE during HIIE to clarify the relationship between OLFE intake and anaerobic metabolism. In addition, d-ROMs and BAP were the only indicators of oxidative stress and antioxidant potential. Future research should examine redox balance using other oxidative stress (e.g., carbonylated protein) and antioxidant potential (e.g., total antioxidants) markers. In addition, we could not measure the respiratory quotient (RQ) during HIIE. Therefore, we discussed anaerobic and aerobic exercise metabolism during HIIE based on previous studies. Investigation of the RQ is required to determine the change in metabolic performance upon OLFE supplementation. These investigations may accurately determine whether OLFE modulates redox balance during high-intensity exercise and promotes skeletal muscle adaptation. Despite having some limitations, our pilot study demonstrated the effects of a seven-day supplementation regimen with $200 \mathrm{mg}$ OLFE on athletic performance. Hence, our study may encourage randomized, placebo-controlled, double-blind, crossover trials to assess the effects of OLFE supplementation on athletic performance in the future.

Finally, we recruited trained athletes from a university sports club who regularly exercised. Optimal OLFE intake depends on ROS production and antioxidant capacity, which are affected by individual conditioning and fitness levels. 
Therefore, OLFE intake should be adjusted for individuals age, muscle mass, and exercise habits.

In conclusion, the present study demonstrated that supplementation with a highly bioavailable polyphenol, OLFE, for seven days at $200 \mathrm{mg}$ /day improves power output during HIIE without adverse effects on post-exercise performance. Therefore, HIIE performance, which requires both anaerobic and aerobic metabolism, may be improved through the antioxidant effect or activation of metabolic factors exerted by short-term OLFE loading. This strategy may contribute to the maintenance of athletic performance, especially when training intensity is increased.

\section{ACKNOWLEDGEMENTS}

This research was funded by Amino Up Co., Ltd. (No. R019-044). The authors would like to thank the members of our laboratory for their technical assistance and the participants belonging to the sports clubs of Nippon Sport Science University for their cooperation in the completion of this study. Masaaki Sugita conducted this study with scholarship donations and test supplements provided by Amino Up Co., Ltd. The funders had no role in the study design, in the collection, analyses, or interpretation of data, in the writing of the manuscript, or in the decision to publish the results.

\section{REFERENCES}

1. Scheffer DL, Silva LA, Tromm CB, da Rosa GL, Silveira PC, de Souza CT, Latini A, Pinho RA. Impact of different resistance training protocols on muscular oxidative stress parameters. Appl Physiol Nutr Metab. 2012;37:1239-46.

2. Hawley JA, Burke LM, Phillips SM, Spriet LL. Nutritional modulation of training-induced skeletal muscle adaptations. J Appl Physiol. 2011;110:834-45.

3. Di Meo S, Napolitano G, Venditti P. Mediators of physical activity protection against ROS-linked skeletal muscle damage. Int J Mol Sci. 2019;20:3024-61.

4. Reid MB. Redox interventions to increase exercise performance. $J$ Physiol. 2016;594:5125-33.

5. Reid MB. Invited review: redox modulation of skeletal muscle contraction: what we know and what we don't. J Appl Physiol. 2001;90:724-31.

6. Moopanar TR, Allen DG. Reactive oxygen species reduce myofibrillar $\mathrm{Ca} 2+$ sensitivity in fatiguing mouse skeletal muscle at 37 degrees C. J Physiol. 2005;564:189-99.

7. Matsunaga S, Inashima S, Yamada T, Watanabe H, Hazama T, Wada M. Oxidation of sarcoplasmic reticulum $\mathrm{Ca}(2+)$-ATPase induced by high-intensity exercise. Pflugers Archiv. 2003;446:394-9.

8. Groussard C, Rannou-Bekono F, Machefer G, Chevanne M, Vincent S, Sergent O, Cillard J, Gratas-Delamarche A. Changes in blood lipid peroxidation markers and antioxidants after a single sprint anaerobic exercise. Eur J Appl Physiol. 2003;89:14-20.

9. Hübner-Woźniak E, Lutosławska G, Panczenko-Kresowska B, Sitkowski D. The effect of oxygen uptake at anaerobic threshold on resting plasma concentrations of reduced glutathione and thiobar- bituric acid reactive substances (TBARS), and on the antioxidant enzyme activities in blood. Biol Sport. 2005;22:151-61.

10. Aoi W, Naito $Y$, Takanami $Y$, Kawai $Y$, Sakuma K, Ichikawa H, Yoshida N, Yoshikawa T. Oxidative stress and delayed-onset muscle damage after exercise. Free Radic Biol Med. 2004;37:480-7.

11. Fatouros IG, Chatzinikolaou A, Douroudos I, Nikolaidis MG, Kyparos A, Margonis K, Michailidis Y, Vantarakis A, Taxildaris K, Katrabasas I, Mandalidis D, Kouretas D, Jamurtas AZ. Timecourse of changes in oxidative stress and antioxidant status responses following a soccer game. J Strength Cond Res. 2010;24:3278-86.

12. Mason SA, Trewin AJ, Parker L, Wadley GD. Antioxidant supplements and endurance exercise: current evidence and mechanistic insights. Redox Biol. 2020;35:101471.

13. Paulsen $\mathrm{G}$, Hamarsland $\mathrm{H}$, Cumming $\mathrm{K}$, Johansen R, Hulmi J, Børsheim E, Wiig H, Garthe I, Raastad T. Vitamin C and E supplementation alters protein signalling after a strength training session, but not muscle growth during 10 weeks of training. J Physiol. 2014;592:5391-408.

14. Theodorou AA, Nikolaidis MG, Paschalis V, Koutsias S, Panayiotou G, Fatouros IG, Koutedakis Y, Jamurtas AZ. No effect of antioxidant supplementation on muscle performance and blood redox status adaptations to eccentric training. The Am J Clin Nutr. 2011;93:1373-83.

15. Bryer SC, Goldfarb AH. Effect of high dose vitamin C supplementation on muscle soreness, damage, function, and oxidative stress to eccentric exercise. Int J Sport Nutr Exerc Metab. 2006;16:27080.

16. Clifford T, Jeffries O, Stevenson EJ, Davies KAB. The effects of vitamin $C$ and $E$ on exercise-induced physiological adaptations: a systematic review and meta-analysis of randomized controlled trials. Crit Rev Food Sci Nutr. 2020;60:3669-79.

17. Kawamura A, Aoi W, Abe R, Kobayashi Y, Kuwahata M, Higashi A. Astaxanthin-, $\beta$-carotene-, and resveratrol-rich foods support resistance training-induced adaptation. Antioxidants. 2021;10:11323.

18. Bell PG, Stevenson E, Davison GW, Howatson G. The effects of montmorency tart cherry concentrate supplementation on recovery following prolonged, intermittent exercise. Nutrients. 2016;8:44151.

19. Bowtell JL, Sumners DP, Dyer A, Fox P, Mileva KN. Montmorency cherry juice reduces muscle damage caused by intensive strength exercise. Med Sci Sports Exerc. 2011;43:1544-51.

20. Connolly DA, McHugh MP, Padilla-Zakour OI, Carlson L, Sayers SP. Efficacy of a tart cherry juice blend in preventing the symptoms of muscle damage. Br J Sports Med. 2006;40:679-83.

21. Howatson G, McHugh MP, Hill JA, Brouner J, Jewell AP, van Someren KA, Shave RE, Howatson SA. Influence of tart cherry juice on indices of recovery following marathon running. Scand $J$ Med Sci Sports. 2010;20:843-52.

22. Machin DR, Christmas KM, Chou TH, Hill SC, Van Pelt DW, Trombold JR, Coyle EF. Effects of differing dosages of pomegranate juice supplementation after eccentric exercise. Physiolo J. 2014;2014:271959.

23. Bowtell J, Kelly V. Fruit-derived polyphenol supplementation for athlete recovery and performance. Sports Med. 2019;49:3-23.

24. Coombes JS, Powers SK, Rowell B, Hamilton KL, Dodd SL, Shanely RA, Sen CK, Packer L. Effects of vitamin E and alpha-li- 
poic acid on skeletal muscle contractile properties. J Appl Physiol. 2001;90:1424-30.

25. Gomez-Cabrera MC, Domenech E, Romagnoli M, Arduini A, Borras C, Pallardo FV, Sastre J, Vina J. Oral administration of vitamin $C$ decreases muscle mitochondrial biogenesis and hampers training-induced adaptations in endurance performance. Am J Clin Nutr. 2008;87:142-9.

26. Di Lorenzo C, Colombo F, Biella S, Stockley C, Restani P. Polyphenols and human health: the role of bioavailability. Nutrients. 2021;13:273-302.

27. Nishioka H, Fujii H, Sun B, Aruoma OI. Comparative efficacy of oligonol, catechin and (-)-epigallocatechin 3-O-gallate in modulating the potassium bromate-induced renal toxicity in rats. Toxicology. 2006;226:181-7.

28. Lee JB, Shin YO, Min YK, Yang HM. The effect of Oligonol intake on cortisol and related cytokines in healthy young men. Nutr Res Pract. 2010;4:203-7.

29. Shin YO, Lee JB, Min YK, Yang HM. Effect of oligonol intake on cortisol and cytokines, and body temperature after leg immersion into hot water. Food Sci Biotechnol. 2011;20:659-63.

30. Noh JS, Park CH, Yokozawa T. Treatment with oligonol, a low-molecular polyphenol derived from lychee fruit, attenuates diabetes-induced hepatic damage through regulation of oxidative stress and lipid metabolism. Br J Nutr. 2011;106:1013-22.

31. Nishizawa M, Hara T, Miura T, Fujita S, Yoshigai E, Ue H, Hayashi Y, Kwon AH, Okumura T, Isaka T. Supplementation with a flavanol-rich lychee fruit extract influences the inflammatory status of young athletes. Phytother Res. 2011;25:1486-93.

32. Tsukamoto-Kusakabe M, Kanbayashi I, Kitadate K, Takayuki I, Kimoto R, Takeda H. Effect of polyphenol converting into a low-molecular form on oxidative stress and serum total antioxidative capacity in high-intensity intermittent spring exercise. Hokkaido J Phys Educ Health Sport Sci. 2011;25:1486-93.

33. Ohno H, Sakurai T, Hisajima T, Abe S, Kizaki T, Ogasawara JE, Ishibashi Y, Imaizumi K, Takemasa T, Haga S, Kitadate K, Nishioka $\mathrm{H}$, Fujii $\mathrm{H}$. The supplementation of oligonol, the new lychee fruit-derived polyphenol converting into a low-molecular form, has a positive effect on fatigue during regular track-and-field training in young athletes. Adv Exerc Sport Physiol. 2008;13:93-9.

34. Gorostiaga EM, Walter CB, Foster C, Hickson RC. Uniqueness of interval and continuous training at the same maintained exercise intensity. Eur J Appl Physiol Occup Physiol. 1991;63:101-7.

35. Tabata I, Irisawa K, Kouzaki M, Nishimura K, Ogita F, Miyachi M. Metabolic profile of high intensity intermittent exercises. Med Sci Sports Exerc. 1997:29:390-5.

36. Sakai K, Sheahan J, Takamatsu K. The relationship between high power output during Intermittent exercise and three energy delivery systems. Jpn J Phys Fitness Sports Med. 1999;48:453-66.

37. lamele L, Fiocchi R, Vernocchi A. Evaluation of an automated spectrophotometric assay for reactive oxygen metabolites in serum. Clin Chem Lab Med. 2002;40:673-6.

38. Nakayama K, Terawaki H, Nakayama M, Iwabuchi M, Sato T, Ito $\mathrm{S}$. Reduction of serum antioxidative capacity during hemodialysis. Clin Exp Nephrol. 2007;11:218-24.

39. Benzie IF, Strain JJ. The ferric reducing ability of plasma (FRAP) as a measure of "antioxidant power": the FRAP assay. Anal Biochem. 1996;239:70-6.

40. Medbo JI, Tabata I. Relative importance of aerobic and anaerobic energy release during short-lasting exhausting bicycle exercise. $J$ Appl Physiol. 1989;67:1881-6.

41. Fick A. Ueber diffusion. Annalen der Physik. 1855;170:59-86

42. Kang SW, Hahn S, Kim JK, Yang SM, Park BJ, Lee SC. Oligomerized lychee fruit extract (OLFE) and a mixture of vitamin $C$ and vitamin $\mathrm{E}$ for endurance capacity in a double blind randomized controlled trial. J Clin Biochem Nutr. 2012;50:106-13.

43. Liu PH, Aoi W, Takami M, Terajima H, Tanimura Y, Naito Y, Itoh Y, Yoshikawa T. The astaxanthin-induced improvement in lipid metabolism during exercise is mediated by a PGC-1alpha increase in skeletal muscle. J Clin Biochem Nutr. 2014;54:86-9.

44. Murase T, Haramizu S, Ota N, Hase T. Suppression of the aging-associated decline in physical performance by a combination of resveratrol intake and habitual exercise in senescence-accelerated mice. Biogerontology. 2009:10:423-34.

45. Brooks GA. The science and translation of lactate shuttle theory. Cell Metab. 2018;27:757-85.

46. Lagouge M, Argmann C, Gerhart-Hines Z, Meziane H, Lerin C, Daussin F, Messadeq N, Milne J, Lambert P, Elliott P. Resveratrol improves mitochondrial function and protects against metabolic disease by activating SIRT1 and PGC-1alpha. Cell. 2006;127:1109-22.

47. Parker L, McGuckin TA, Leicht AS. Influence of exercise intensity on systemic oxidative stress and antioxidant capacity. Clin Physiol Funct Imaging. 2014;34:377-83

48. Arent SM, Senso M, Golem DL, McKeever KH. The effects of theaflavin-enriched black tea extract on muscle soreness, oxidative stress, inflammation, and endocrine responses to acute anaerobic interval training: a randomized, double-blind, crossover study. J Int Soc Sports Nutr. 2010;7:11-20.

49. Keane KM, Bailey SJ, Vanhatalo A, Jones AM, Howatson G. Effects of montmorency tart cherry (L. Prunus Cerasus) consumption on nitric oxide biomarkers and exercise performance. Scand $J$ Med Sci Sports. 2018;28:1746-56

50. Bianchi CP, Shanes A. Calcium influx in skeletal muscle at rest, during activity, and during potassium contracture. J Gen Physiol. 1959;42:803-15 